Tropical Journal of Pharmaceutical Research August 2020; 19 (8): 1661-1668

ISSN: $1596-5996$ (print); 1596-9827 (electronic)

(C) Pharmacotherapy Group, Faculty of Pharmacy, University of Benin, Benin City, 300001 Nigeria

\title{
Suppressive efficiency of Kojic acid from Aspergillus tamarii MM11 against HepG-2 cell line derived from human liver cancer
}

\author{
Mohammad M El-Metwally', Eman R ElBealy², Doha M Beltagy³, Mohamed \\ Shaaban ${ }^{4}$, Attalla F El-kott ${ }^{5,6 *}$ \\ ${ }^{1}$ Botany and Microbiology Department, Faculty of Science, Damanhour University, Damanhour, Egypt, ${ }^{2}$ Biology Department, \\ College of Science for girls, King Khalid University, Abha, Saudi Arabia, ${ }^{3}$ Biochemistry Division, Chemistry Department, Faculty \\ of Science, Damanhour University, Damanhour, ${ }^{4}$ Chemistry of Natural Compounds Department, Pharmaceutical and Drug \\ Industries Research Division, National Research Centre, El-Behoos St., Dokki-Cairo 12622, Egypt, ${ }^{5}$ Department of Biology, \\ College of Science, King Khalid University, Abha, Saudi Arabia, ${ }^{6}$ Zoology Department, College of Science, Damanhour \\ University, Damanhour, Egypt
}

*For correspondence: Email: elkottaf@yahoo.com

Sent for review: 24 January 2020

Revised accepted: 20 July 2020

\begin{abstract}
Purpose: To evaluate the antioxidant and cytotoxic properties of Kojic acid (KOJIC ACID) from Aspergillus tamarii MM11 against HepG-2 cell line derived from human liver cancer.

Methods: The crude extract of $A$. tamarii MM11 was dissolved in a mixture of $\mathrm{CH}_{2} \mathrm{Cl}_{2} / \mathrm{MeOH}(85: 15)$ and separation was done using silica gel chromatography using gradient size exclusion chromatograph. The non-polar oily fractions were subjected to gas chromatography-mass spectrometric (GC-MS) analysis. Kojic acid structure was identified by $x$-beam crystallography and spectroscopic methods. Total antioxidant properties of KOJIC ACID were evaluated by using 1,1-diphenyl-2- picrylhydrazyl (DPPH) against ascorbic acid as a reference. The cytotoxic activity of KOJIC ACID from A. tamarii MM11 was investigated on the human cell line of liver cancer (HepG-2) using a sulforhodamine $B$ (SRB) assay based on a cell density determination by the measurement of cellular protein content.

Result: Highly bioactive Kojic acid was isolated as the main product. A. tamarii MM11 Kojic acid showed good antioxidant activity with half-maximal inhibitory concentration of $I C_{50}$ at concentrations of 10.34 compared to $6.79 \mu \mathrm{g} / \mathrm{mL}$ for ascorbic acid. Kojic acid also showed good cytotoxic activity against HepG-2 cell line of human liver cancer with $I C_{50}$ at 6.20 compared to $3.25 \mu \mathrm{g} / \mathrm{mL}$ of reference drug doxorubicin.

Conclusion: Kojic acid produced naturally from A. tamarii MM11 shows good antioxidant and cytotoxic activity against HepG-2 cell line derived from human liver cancer. These findings suggest that Kojic acid can be therapeutically used as an antitumor drug after further in vivo studies.
\end{abstract}

Keywords: Aspergillus tamarii, Secondary metabolites, Kojic acid, Anticancer, Liver cancer

This is an Open Access article that uses a fund-ing model which does not charge readers or their institutions for access and distributed under the terms of the Creative Commons Attribution License (http://creativecommons.org/licenses/by/4.0) and the Budapest Open Access Initiative (http://www.budapestopenaccessinitiative.org/read), which permit unrestricted use, distribution, and reproduction in any medium, provided the original work is properly credited.

Tropical Journal of Pharmaceutical Research is indexed by Science Citation Index (SciSearch), Scopus, International Pharmaceutical Abstract, Chemical Abstracts, Embase, Index Copernicus, EBSCO, African Index Medicus, JournalSeek, Journal Citation Reports/Science Edition, Directory of Open Access Journals (DOAJ), African Journal Online, Bioline International, Open-J-Gate and Pharmacy Abstracts

\section{INTRODUCTION}

Fungi this huge world produce multiple types of secondary metabolites, which including aromatic compounds, amino acids, anthracenones, butanolides, butenolides, cytochalasans, macrolides, naphthalenones, pyrones, terpenes,

(c) 2020 The authors. This work is licensed under the Creative Commons Attribution 4.0 International License 
etc. [1,2]. These compounds have numerous industrial, ecological and pharmaceutical uses.

Cancer is a life-threatening disease. Most of the successive anticancer medications currently used cause many undesirable side effects. For example, Doxorubicin can prompt cardiotoxicity and tumor drug resistance [3]. Methotrexate also can cause liver damage and portal hypertension and cirrhosis. While, Cisplatin administration can lead to nephrotoxicity and in some cases renal failure [4].

Therefore, new anticancer drugs with more efficiency and ability to mitigate side effects are needed. Fungal anticancer secondary metabolites are one of the very important targets for mycologist. In this connection, 5-hydroxy-2hydroxymethyl-y-pyrone (HMP) or Kojic acid (KOJIC ACID) is a major secondary metabolite produced by a limited range of microorganisms, including Aspergillus oryzae, $A$. flavus, and $A$. tamarii, as well as Penicillium species and certain bacteria [5].

Kojic acid possess strong antioxidant, antibacterial and antifungal activities. So, it is widely used in medical purposes and many other fields. It also used as a food flavor enhancer [6]. In agriculture, it used as anti-melanosis and insecticide activator [7]. In cosmetic, Kojic acid is well known as whitening agent, ultraviolet filter, tyrosinase inhibitor, and radio-protective agent [8]. Few studies were performed on anticancer activity of Kojic acid.

In the present investigation, most of the secondary metabolome of terrestrial $A$. tamarii MM11 was detected and the produced Kojic acid was isolated, purified and elucidated and its antioxidant properties were studied. Furthermore, the cytotoxic effect of Kojic acid on liver carcinoma cell lines were determined.

\section{EXPERIMENTAL}

\section{Fungal strain}

The terrestrial fungal isolate used in this study was isolated from tubers of rotten Jerusalem artichoke $(H$. tuberosus) identified by molecular sequencing of fungal Inter Transcribed Spacer (ITS). The fungus strain was identified as $A$. tamarii MM11 with the accession no. GU295949.

\section{Fermentation}

A. tamarii MM11 was inoculated from well grown agar plates colonies in $0.1 \mathrm{~L}$ sterilized glass bottles each containing sterilized rice. The medium composition was: $8 \mathrm{~g}$ commercial rice; $10 \mathrm{~mL}$ distilled water. The bottles were incubated for 15 days at $28 \pm 2{ }^{\circ} \mathrm{C}$. After harvesting, $50 \mathrm{~mL}$ of $1: 1 \mathrm{DCM} / \mathrm{MeOH}$ was added to each bottle, followed by vigorous shaking for two hours. The afforded organic extract was decanted, filtered and then concentrated in vacuo till dryness.

\section{Separation and purification of fungal secondary metabolome}

The crude extract $(7.79 \mathrm{~g})$ was dissolved in a mixture of $\mathrm{CH}_{2} \mathrm{Cl}_{2} / \mathrm{MeOH}$ (85:15). Six gram of silica gel were added and this mixture was brought to dryness under reduced pressure. Separation was performed using a silica gel column (3 x $100 \mathrm{~cm}, 200 \mathrm{~g})$ chromatography

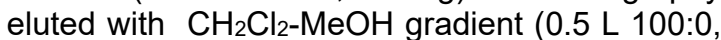
0.5 L 98:2, 0.6 L 95:5, 0.5 L 93:7, 0.6 L 90:10, $0.3 \mathrm{~L}$ 80:20 and 0.3 L 50:50 v.v). After TLC monitoring, four fractions were afforded, FI (3.2 g), FII (1.2 g), FIII (3.2 g). Fraction I (3.2 g) was then applied to purification on Sephadex $\mathrm{LH}-20$ $\left(\mathrm{CH}_{2} \mathrm{Cl}_{2}-\mathrm{MeOH}, 60: 40\right)$ affording a large oily zone of fraction1 $(2.1 \mathrm{~g})$, which on application to GCMS analysis afforded seventy-three compounds, including naphthalene (1), 1-methyl-naphthalene (3), 2,7-dimethyl-naphthalene (9), 1,3-dimethylnaphthalene (10), 2,6-dimethyl-naphthalene (11) and penta-chloro-pyridine (14).

Fraction II (1.2 g) was purified on Sephadex LH$20\left(\mathrm{CH}_{2} \mathrm{Cl}_{2}-\mathrm{MeOH}, 60: 40\right)$, yielding a large zone of oily mixture $(0.9 \mathrm{~g})$, which onapplication to GCMS analysis delivered twenty-six compounds, including ethyl-2-methyl-3-Oxo-hexanoate (71), 1,2-dibromo-2-methyl-propane (72), azulene (75), bicyclo [4.3.0] nonane, 3-butyl-4-hexyl- (83), 6-nitroundec-5-ENE (84), 4,9-decadienoic acid, 2-nitro-, ethyl ester (88) and 1-chloro-octadecane (89). The last fraction FIII (3.2 g) applied to washing with dichloromethane followed by filtration to afford colourless needles of Kojic acid $(1,2.3 \mathrm{~g})$.

The nuclear magnetic resonance (NMR) spectra were determined on Varian Unity 300 (300.145 $\mathrm{MHz}$ ) and Varian Inova 600 (150.820 MHz) spectrometers. Electro spray ionization mass spectra (ESI MS) was recorded on a Finnigan LCQ with quaternary pump Rheos 4000 (Flux Instrument). Flash chromatography was carried out on silica gel (230-400 mesh).

The rate of flow $\left(R_{\mathrm{f}}\right.$ ) values were measured on Polygram SIL G/UV 254 TLC cards (MachereyNagel \& Co.). Size exclusion chromatography was done on Sephadex LH-20 (Lipophilic Sephadex, Amersham Biosciences Ltd; purchased from Sigma-Aldrich Chemie, 
Steinheim, Germany). Mass spectra were recorded on a Finnigan MAT 95 spectrometer $(70 \mathrm{eV})$. All solvents and chemicals were purchased from Sigma, Merck and Aldrich.

\section{Determination of total antioxidant activity}

The antioxidant activities of fungal KOJIC ACID were detected using 1,1-diphenyl-2picrylhydrazyl (DPPH) in comparison with ascorbic acid as standard radical scavenging agent. The experiment was carried out by preparing solutions of $50 \mathrm{mg} / \mathrm{mL}$, and then serial dilutions $(5-50 \mathrm{mg} / \mathrm{mL})$ of KOJIC ACID and the reference ascorbic acid were prepared. Then, $250 \mu \mathrm{L}$ of each dilution was added to $1 \mathrm{~mL}$ $\mathrm{DPPH}$ solution $(6 \mathrm{mg} / 50 \mathrm{~mL})$. Control tube was also prepared using $1 \mathrm{~mL}$ of ethanol. The mixture was shaken and incubated for $30 \mathrm{~min}$ in the dark at room temperature. Absorbance was measured using a Genway spectrophotometer at $517 \mathrm{~nm}$ [9]. These steps were repeated 3 times and the radical scavenging $(R)$ evaluated according to Eq 1.

$R \%=1-\left(A_{s} / A_{c}\right) \times 100$

where, $R$ is radical scavenging, $A_{s}$ is the absorbance of the sample and $A_{c}$ is the absorbance of the control.

\section{Evaluation of cytotoxic activity}

The cytotoxic activity of KOJIC ACID of $A$. tamarii MM11 was investigated on the human cell line of liver cancer (HepG-2) using sulforhodamine B (SRB) assay based on a cell density determination by the measurement of cellular protein content. HepG-2 monolayer was fixed on the 96-well plate with trichloroacetic acid (TCA). Then, SRB was added to each well and incubated at room temperature for $1 \mathrm{~h}$. SRB binds to basic amino acids in cellular proteins under mild acidic conditions. The excess dye was removed by washing repeatedly with acetic acid. The protein bound dye was dissolved by adding Tris-base solution (basic medium) to each well and shake the plate to solubilize the protein bound dye.

The amount of bound dye can be determined by measuring the absorbance at $510 \mathrm{~nm}$ in a microplate reader. It can then be extrapolated to measure cell proliferation [10]. HepG-2 used in this study was obtained from the American Type Culture Collection (ATCC, Minisota, U.S.A.). The tumor cell line was maintained at the National Cancer Institute, Cairo, Egypt, through serial sub-culturing. Doxorubicin was used as the reference drug.

\section{Statistical analysis}

Statistical analysis of the results was carried out using GraphPad instant, Version 3.06 (GraphPad Software Inc, San Diego, California, USA). The data are expressed as mean \pm standard deviation (SD). Curves plotting were performed with Origin 6.0

\section{RESULTS}

\section{Phytochemical profile}

\section{Non-polar fractions}

GC-MS analysis of the un-polar oily fraction of fraction I (Figure 2) afforded the shown below listed of compounds (Table 1). In accordance, seventy three compounds were identified namely, naphthalene (2), n-dodecane (3), 1methyl-napthalene (4), n-tridecane (5), 3-methylhexadecane (6), 1,1'-biphenyl (7), 7-tetradecene (8), tetradecane (9), 2,7-dimethyl-napthalene (10), 1,3-dimethyl-napthalene (11), 2,6-dimethylnapthalene (12), n-dotriacontane (13), pentadecane (14), penta-chloro-pyridine (15), 7methyl-pentadecane (16), 5-methyl-decane (17), 3-methyl-eicosane (18), 9h-fluorene (19), 1hexadecene (20), hexadecane (21), 7hexadecene (22), 1-hexyl-3-methyl-cyclopentane (23), butyl-tridecyl-sulfurous acid ester (24), 4,6dimethyl-dodecane (25), 1,3-dibutenyl-4-phenylbenzene (26), 5-methyl-tridecane (27), phensuximide (28), phenanthrene (29), e-15heptadecenal (30), octadecane (31), pentacosane (32), (2-propyl) octadecyl sulfurous acid (33), nonadecane (34), allyl-tridecyl-oxalate (35), methyl-hexadecanoate (36), 10-methylnonadecane (37), 2-ethylhexylheptadecyl sulfurous acid (38), dodecyl-hexyl-oxalate (39), hexyl-tetradecyl-oxalate (40), ethylhexadecanoate (41), eicosane, 5-methyl-1-heptene (42), 1,2-dibromo-dodecane (43), 2-chloroethyllinoleate (44), methyl-10-methyl-heptadecanoate (45), 3-decen-1-ol (46), 9,12-octadecadienoic acid $(z, z)(47)$, 12-methyl-e,e-2,13-octadien-1-ol (48), pentadecyl-3-bromo-benzoate (49), ethyllinoleate, ethyloleate, methyl-17-methyloctadecanoate (50), 1,13-tetradecadien-3-one (51), n-[4-bromo-n-butyl]-2-piperidinone (52), 3ethyl-1-octene (53), 3,4-dimethyl-heptane (54), 2fluoro-1-triacetylribofuranosyl-imidazole (55), 1fluoro-tetradecane (56), 1,2-epoxy-hexadecane (57), 7-hexadecyne (58), 2-tridecyloxirane (59), trans-cinnamonitrile (60), (9e)-9-hexacosene (61), dotriacontane (62), 3-(2,5-dimethyl-1hpyrrole-3-yl)-1,3-dihydro-indol-2-one (63), 1(dodecyloxy)-2,3-epoxy- propane (64), 1bromopentadecane (65), squalene (66), tetradecanal (67), Sulfurous acid, butyl 
heptadecyl ester (68), 2-(4-hydroxybutyl)-2nitrocyclodecanone vinylcyclododecane (71).
(69), 1,2-Epoxy-1(70), 17-Pentatriacontene
Alternatively, an analysis of the un-polar oily subfraction by GC-MS (Fig. 3) established the existence of twenty six metabolic compounds (Table 2), namely: Ethyl-2-methyl-3-oxohexanoate (72), 1,2-dibromo-2-methyl-propane (73), benzeneacetic acid, methyl ester (74), Azulene (75), n-tetradecane (76), pentadecanoic acid, ethyl ester (77), Hexadecanoic acid, methyl ester, hexadecanoic acid, ethyl ester, 10,13octadecadienoic acid, methyl ester (78), octadecanoic acid, methyl ester (79), heptadecanoic acid, 16-methyl-, methyl ester (80), ethyl linoleate (81), 9-octadecenoic acid (z), ethyl ester (82), bicyclo[4.3.0]nonane, 3-butyl-4hexyl- (83), 6-nitroundec-5-ene (84), 1,2benzenedicarboxylic acid, bis (2-ethylhexyl) ester (85), di-n-octyl phthalate (86), 1,2benzenedicarboxylic acid, diisooctyl ester (87), 4,9-decadienoic acid, 2-nitro-, ethyl ester (88), octadecane, 1-chloro- (89), 1,2benzenedicarboxylic acid, diisononyl ester, phthalic acid, nonyl tridec-2-yn-1-yl ester (90), didodecyl phthalate.

As colourless needles, kojic acid (KOJIC ACID) (1) was afforded from the polar fraction FIII after washing with hot dichloromethane. The structure of KOJIC ACID (1) was definitely deduced on the basis of X-ray crystallography and spectroscopic means. Kojic acid (1): $\mathrm{C}_{6} \mathrm{H}_{6} \mathrm{O}_{4}$ (142). UV absorbing, colorless solid, $R_{\mathrm{f}}=0.15\left(\mathrm{CHCl}_{3} / 10 \%\right.$ $\mathrm{MeOH})$, turned blue on spraying with anisaldehyde/sulfuric after heating. - ${ }^{1} \mathrm{HNMR}$ (DMSO-d6, $300 \mathrm{MHz}$ ): $\delta=8.99$ (brs, 1H, OH), 7.97 (s 1H, CH-2), 6.34 (s, 1H, CH-5), 5.69 (brs, $1 \mathrm{H}, \mathrm{OH}), 4.28\left(\mathrm{~s}, 2 \mathrm{H}, \mathrm{CH}_{2}-7\right) .{ }^{-13} \mathrm{C} / \mathrm{APT}$ NMR (DMSO-d6, $125 \mathrm{MHz}) \delta=174.2\left(\mathrm{C}_{\mathrm{q}^{-}}-4\right), 168.2\left(\mathrm{C}_{\mathrm{q}^{-}}\right.$ 6), $153.0\left(\mathrm{C}_{\mathrm{q}}-3\right), 138.9(\mathrm{CH}-2), 110.0(\mathrm{CH}-5)$, $59.7\left(\mathrm{CH}_{2}-7\right)$. -EI MS m/z (\%): $142([\mathrm{M+}], 100$.$) ,$ $113([\mathrm{M}-\mathrm{CHO}]+, 33), 97$ (15), 85 (18), 69 (74), 57 (25), 39 (42), 29 (52).

The following data for the unit cell were obtained from X-ray oscillation photographs: $a=3 \cdot 85, b=$ $18 \cdot 4, c=8.84$ A.; $\beta=74^{\circ}$, correct to about \pm 1 per cent as typical to those reported one. The measured density of $1.58 \mathrm{~g}$. cm-3 gave $3.98 \sim 4$ molecules/cell. These results are in accord with previous measurements [11,12].

According to EI MS, the molecular weight of Kojic acid was established as 142 Dalton with a corresponding molecular formula of $\mathrm{C}_{6} \mathrm{H}_{6} \mathrm{O}_{4}$. Based on the proton nuclear magnetic resonance spectroscopy ( ${ }^{1} \mathrm{H}$ NMR, DMSO-d6), two singlets were visible at 7.97 and 6.34, being for aromatic/olefinic attached protons, together with two broad singlets at $\delta 8.99$ and 5.69 ppm being for phenolic and aliphatic hydroxyl protons, respectively, in addition to an $s p^{2}$-attached oxymethylene protons were shown at $\delta 4.28 \mathrm{ppm}$. On the bases of ${ }^{13} \mathrm{C}$ NMR/APT spectra of Kojic acid, six carbon signals, as matched with the afforded molecular formula, were deduced, being for one $\mathrm{y}$-lactone carbonyl ( $\delta$ 174.2), three $s p^{2}$ quaternary Oxy-carbons ( $\delta 168.2$ and 153.0), two $s p^{2}-\mathrm{CH}$ carbon signals $(\delta 138.9$ and 110.0$)$ and one $\quad s p^{2}$-attached Oxy-methylene (59.7). According to these data, searching in AntiBase and comparison with literature, Kojic acid structure was confirmed.

Kojic acid from $A$. tamarii MM11 recorded strong antioxidant activities with $\mathrm{IC}_{50}$ value reached to $10.34 \mu \mathrm{g} / \mathrm{mL}$ in comparison with the reference compound (ascorbic acid) which recorded $\mathrm{IC}_{50}$ of $6.79 \mu \mathrm{g} / \mathrm{mL}$. These values indicated potent radical scavenging activities of KOJIC ACID (Table 3). Kojic acid also showed excellent cytotoxic activities against cancerous human liver cell line (HepG-2) with IC 50 equals to $6.20 \mu \mathrm{g} / \mathrm{mL}$ in comparison with $3.25 \mu \mathrm{g} / \mathrm{mL}$ for the reference drug Doxorubicin (DOX) (Figure 6)

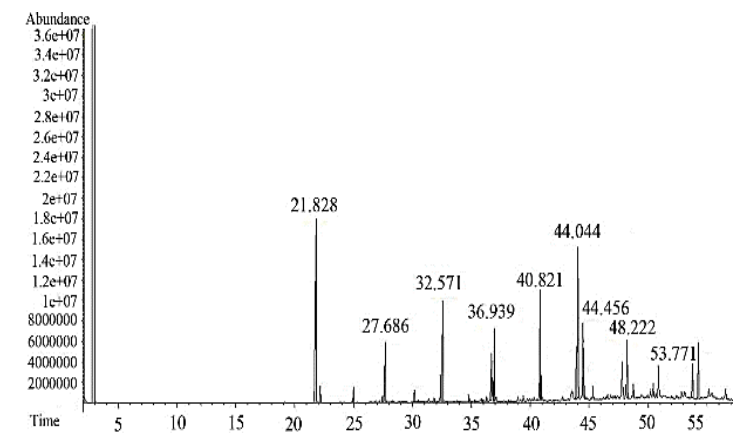

Figure 1: GC-MS chromatogram of oily fraction of fraction I

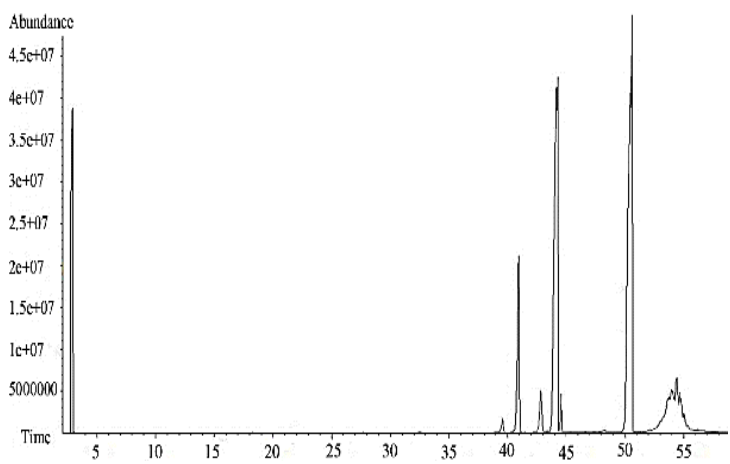

Figure 2: GC-MS chromatogram of fraction II

Trop J Pharm Res, August 2020; 19(8): 1664 
Table 1: Compounds detected in oily fraction I by GC-MS

\begin{tabular}{|c|c|c|c|c|c|}
\hline No. & Name & MF* $^{*}$ & MWt & $R_{T}$ & $\mathbf{A} \%$ \\
\hline 1 & Naphthalene (2) & $\mathrm{C}_{10} \mathrm{H}_{8}$ & 128 & 21.83 & 11.03 \\
\hline 2 & n-Dodecane (3) & $\mathrm{C}_{12} \mathrm{H}_{26}$ & 170 & 22.18 & 0.38 \\
\hline 3 & 1-Methyl-napthalene (4) & $\mathrm{C}_{11} \mathrm{H}_{10}$ & 142 & 24.89 & 0.06 \\
\hline 4 & n-Tridecane (5) & $\mathrm{C}_{13} \mathrm{H}_{28}$ & 184 & 25.01 & 0.40 \\
\hline 5 & 3-Methyl-hexadecane (6) & $\mathrm{C}_{17} \mathrm{H}_{36}$ & 240 & 26.88 & 0.05 \\
\hline 6 & 1,1'-Biphenyl (7) & $\mathrm{C}_{12} \mathrm{H}_{10}$ & 154 & 27.18 & 0.04 \\
\hline 7 & 7-Tetradecene (8) & $\mathrm{C}_{14} \mathrm{H}_{28}$ & 196 & 27.45 & 0.20 \\
\hline 8 & Tetradecane (9) & $\mathrm{C}_{14} \mathrm{H}_{30}$ & 198 & 27.69 & 1.66 \\
\hline 9 & 2,7-Dimethyl-napthalene (10) & $\mathrm{C}_{12} \mathrm{H}_{12}$ & 156 & 27.85 & 0.1 \\
\hline 10 & 1,3-Dimethyl-napthalene (11) & $\mathrm{C}_{12} \mathrm{H}_{12}$ & 156 & 28.24 & 0.05 \\
\hline 11 & 2,6-Dimethyl-napthalene (12) & $\mathrm{C}_{12} \mathrm{H}_{12}$ & 156 & 28.32 & 0.06 \\
\hline 12 & n-Dotriacontane (13) & $\mathrm{C}_{32} \mathrm{H}_{66}$ & 450 & 29.23 & 0.05 \\
\hline 13 & Pentadecane (14) & $\mathrm{C}_{15} \mathrm{H}_{32}$ & 212 & 30.15 & 0.33 \\
\hline 14 & Penta-chloro-pyridine (15) & $\mathrm{C}_{5} \mathrm{Cl}_{5} \mathrm{~N}$ & 249 & 30.46 & 0.08 \\
\hline 15 & 7-Methyl-pentadecane (16) & $\mathrm{C}_{16} \mathrm{H}_{34}$ & 226 & 31.26 & 0.08 \\
\hline 16 & 5-Methyl-decane (17) & $\mathrm{C}_{11} \mathrm{H}_{24}$ & 156 & 31.39 & 0.13 \\
\hline 17 & 3-mMethyl-eicosane (18) & $\mathrm{C}_{21} \mathrm{H}_{44}$ & 266 & 31.83 & 0.16 \\
\hline 18 & 9H-Fluorene (19) & $\mathrm{C}_{13} \mathrm{H}_{10}$ & 166 & 32.22 & 0.04 \\
\hline 19 & 1-Hexadecene $(\mathbf{2 0})$ & $\mathrm{C}_{16} \mathrm{H}_{32}$ & 224 & 32.37 & 0.88 \\
\hline 20 & Hexadecane (21) & $\mathrm{C}_{16} \mathrm{H}_{34}$ & 226 & 32.57 & 2.90 \\
\hline 21 & 7-Hexadecene (22) & $\mathrm{C}_{16} \mathrm{H}_{32}$ & 224 & 32.64 & 0.01 \\
\hline 22 & 1-Hexyl-3-methyl-cyclopentane (23) & $\mathrm{C}_{12} \mathrm{H}_{24}$ & 168 & 33.79 & 0.05 \\
\hline 23 & Butyl-tridecyl-sulfurous acid ester (24) & $\mathrm{C}_{17} \mathrm{H}_{36} \mathrm{O}_{3} \mathrm{~S}$ & 320 & 33.94 & 0.03 \\
\hline 24 & 4,6-Dimethyl-dodecane (25) & $\mathrm{C}_{14} \mathrm{H}_{30}$ & 198 & 34.88 & 0.13 \\
\hline 25 & 1,3-Dibutenyl-4-phenyl-benzene (26) & $\mathrm{C}_{20} \mathrm{H}_{22}$ & 262 & 35.30 & 0.08 \\
\hline 26 & 5-methyl-tridecane (27) & $\mathrm{C}_{14} \mathrm{H}_{30}$ & 198 & 35.88 & 0.11 \\
\hline 27 & Phensuximide (28) & $\mathrm{C}_{11} \mathrm{H}_{11} \mathrm{NO}_{2}$ & 189 & 36.01 & 0.11 \\
\hline 28 & Phenanthrene (29) & $\mathrm{C}_{14} \mathrm{H}_{10}$ & 178 & 36.68 & 2.09 \\
\hline 29 & E-15-Heptadecenal (30) & $\mathrm{C}_{17} \mathrm{H}_{34}$ & 238 & 36.78 & 1.31 \\
\hline 30 & Octadecane (31) & $\mathrm{C}_{18} \mathrm{H}_{38}$ & 254 & 36.94 & 1.96 \\
\hline 31 & Pentacosane (32) & $\mathrm{C}_{25} \mathrm{H}_{52}$ & 352 & 37.09 & 0.22 \\
\hline 32 & (2-propyl) octadecyl sulfurous acid (33) & $\mathrm{C}_{21} \mathrm{H}_{44} \mathrm{O}_{3} \mathrm{~S}$ & 376 & 38.17 & 0.08 \\
\hline 33 & Nonadecane (34) & $\mathrm{C}_{19} \mathrm{H}_{40}$ & 268 & 38.92 & 0.16 \\
\hline 34 & Allyl-tridecyl-oxalate (35) & $\mathrm{C}_{18} \mathrm{H}_{32} \mathrm{O}_{4}$ & 312 & 39.15 & 0.12 \\
\hline 35 & Methyl-hexadecanoate (36) & $\mathrm{C}_{17} \mathrm{H}_{34} \mathrm{O}_{2}$ & 270 & 39.44 & 0.09 \\
\hline 36 & 10-methyl-nonadecane (37) & $\mathrm{C}_{20} \mathrm{H}_{42}$ & 280 & 39.72 & 0.08 \\
\hline 37 & 2-ethylhexylheptadecyl sulfurous acid (38) & $\mathrm{C}_{25} \mathrm{H}_{52} \mathrm{O}_{3} \mathrm{~S}$ & 432 & 39.77 & 0.11 \\
\hline 38 & Dodecyl-hexyl-oxalate (39) & $\mathrm{C}_{20} \mathrm{H}_{38} \mathrm{O}_{4}$ & 342 & 39.92 & 0.09 \\
\hline 39 & Hexyl-tetradecyl-oxalate (40) & $\mathrm{C}_{22} \mathrm{H}_{42} \mathrm{O}_{4}$ & 370 & 40.18 & 0.17 \\
\hline 40 & Ethylhexadecanoate (41) & $\mathrm{C}_{18} \mathrm{H}_{36} \mathrm{O}_{2}$ & 284 & 40.82 & 4.63 \\
\hline 41 & Eicosane & $\mathrm{C}_{20} \mathrm{H}_{42}$ & 282 & 40.91 & 1.17 \\
\hline 42 & 5-Methyl-1-heptene (42) & $\mathrm{C}_{8} \mathrm{H}_{16}$ & 112 & 41.04 & 0.10 \\
\hline 43 & 1,2-Dibromo-dodecane (43) & $\mathrm{C}_{12} \mathrm{H}_{24} \mathrm{Br}_{2}$ & 328 & 41.59 & 0.05 \\
\hline 44 & 2-Chloroethyl-linoleate (44) & $\mathrm{C}_{20} \mathrm{H}_{35} \mathrm{ClO}_{2}$ & 341 & 42.64 & 0.09 \\
\hline 45 & Methyl-10-methyl-heptadecanoate (45) & $\mathrm{C}_{19} \mathrm{H}_{38} \mathrm{O}_{2}$ & 298 & 43.21 & 0.11 \\
\hline 46 & 3-Decen-1-ol (46) & $\mathrm{C}_{10} \mathrm{H}_{20} \mathrm{O}$ & 156 & 43.42 & 0.33 \\
\hline 47 & 9,12-octadecadienoic acid $(\mathrm{Z}, \mathrm{Z})(\mathbf{4 7})$ & $\mathrm{C}_{18} \mathrm{H}_{32} \mathrm{O}_{2}$ & 280 & 43.51 & 0.52 \\
\hline 48 & 12-Methyl-E,E-2,13-octadien-1-ol (48) & $\mathrm{C}_{19} \mathrm{H}_{36} \mathrm{O}$ & 280 & 43.55 & 0.09 \\
\hline 49 & Pentadecyl-3-bromo-benzoate (49) & $\mathrm{C}_{22} \mathrm{H}_{35} \mathrm{BrO}_{2}$ & 280 & 43.60 & 0.55 \\
\hline 50 & Ethyllinoleate & $\mathrm{C}_{20} \mathrm{H}_{36} \mathrm{O}_{2}$ & 308 & 43.90 & 2.07 \\
\hline 51 & Ethyloleate & $\mathrm{C}_{20} \mathrm{H}_{38} \mathrm{O}_{2}$ & 310 & 44.04 & 6.31 \\
\hline 52 & Methyl-17-methyl-octadecanoate (50) & $\mathrm{C}_{20} \mathrm{H}_{40} \mathrm{O}_{2}$ & 312 & 44.46 & 3.12 \\
\hline 53 & 1,13 -Tetradecadien-3-one (51) & $\mathrm{C}_{14} \mathrm{H}_{24} \mathrm{O}$ & 208 & 44.68 & 0.16 \\
\hline 54 & N-[4-bromo-n-butyl]-2-piperidinone (52) & $\mathrm{C}_{9} \mathrm{H}_{16} \mathrm{BrNO}$ & 234 & 45.14 & 0.10 \\
\hline 55 & 3-Ethyl-1-octene (53) & $\mathrm{C}_{10} \mathrm{H}_{20}$ & 140 & 45.31 & 0.45 \\
\hline 56 & 3,4-Dimethyl-heptane (54) & $\mathrm{C}_{9} \mathrm{H}_{20}$ & 128 & 45.37 & 0.10 \\
\hline 57 & 2-Fluoro-1-triacetylribofuranosyl-imidazole (55) & $\mathrm{C}_{11} \mathrm{H}_{17} \mathrm{FN}_{2} \mathrm{O}_{7}$ & 344 & 46.19 & 0.10 \\
\hline 58 & 1-Fluoro-tetradecane $(\mathbf{5 6})$ & $\mathrm{C}_{14} \mathrm{H}_{29} \mathrm{~F}$ & 344 & 46.33 & 0.04 \\
\hline 59 & 1,2-epoxy-hexadecane (57) & $\mathrm{C}_{16} \mathrm{H}_{32} \mathrm{O}$ & 240 & 46.45 & 0.12 \\
\hline 60 & 7-Hexadecyne (58) & $\mathrm{C}_{16} \mathrm{H}_{30}$ & 222 & 48.14 & 0.77 \\
\hline 61 & 2-Tridecyloxirane (59) & $\mathrm{C}_{13} \mathrm{H}_{26} \mathrm{O}$ & 198 & 49.50 & 0.77 \\
\hline 62 & Trans-Cinnamonitrile $(60)$ & $\mathrm{C}_{9} \mathrm{H}_{7} \mathrm{~N}$ & 129 & 50.43 & 0.66 \\
\hline 63 & (9E)-9-Hexacosene (61) & $\mathrm{C}_{26} \mathrm{H}_{52}$ & 364 & 50.88 & 1.33 \\
\hline 64 & Dotriacontane $(62)$ & $\mathrm{C}_{32} \mathrm{H}_{66}$ & 450 & 50.93 & 0.43 \\
\hline
\end{tabular}




\begin{tabular}{llcccc}
\hline 65 & 3-(2,5-Dimethyl-1H-pyrrole-3-yl)-1,3-dihydro-indol- & $\mathrm{C}_{14} \mathrm{H}_{14} \mathrm{~N}_{2} \mathrm{O}$ & 226 & 51.75 & 0.15 \\
& 2-one (63) & $\mathrm{C}_{16} \mathrm{H}_{32} \mathrm{O}_{2}$ & 256 & 52.46 & 0.12 \\
66 & 1-(dodecyloxy)-2,3-epoxy- Propane (64) & $\mathrm{C}_{16} \mathrm{H}_{31} \mathrm{Br}$ & 291 & 52.85 & 0.45 \\
67 & 1-Bromopentadecane (65) & $\mathrm{C}_{30} \mathrm{H}_{50}$ & 410 & 54.26 & 1.54 \\
68 & Squalene (66) & $\mathrm{C}_{14} \mathrm{H}_{28} \mathrm{O}$ & 212 & 54.49 & 0.11 \\
69 & Tetradecanal (67) & $\mathrm{C}_{21} \mathrm{H}_{44} \mathrm{O}$ & 312 & 55.14 & 0.49 \\
70 & Sulfurous acid, butyl heptadecyl ester (68) & $\mathrm{C}_{14} \mathrm{H}_{25} \mathrm{NO}_{4}$ & 271 & 55.30 & 0.11 \\
71 & 2-(4-hydroxybutyl)-2-nitrocyclodecanone (69) & $\mathrm{C}_{14} \mathrm{H}_{24} \mathrm{O}$ & 208 & 56.45 & 0.12 \\
72 & 1,2-Epoxy-1-vinylcyclododecane (70) & $\mathrm{C}_{35} \mathrm{H}_{70}$ & 490 & 56.57 & 0.63 \\
73 & 17-Pentatriacontene (71) &
\end{tabular}

${ }^{*} \mathrm{MF}=$ molecular formula; Mwt = molecular weight; Rt = retention time; $\mathrm{A} \%=$ abundance

Table 2: Compounds detected in fraction II by GC-MS

\begin{tabular}{llcccc}
\hline No. & Name & $\mathbf{M F}$ & $\mathbf{M W t}$ & $\boldsymbol{R} \mathbf{T}$ & $\mathbf{A} \%$ \\
\hline 1 & Ethyl-2-methyl-3-oxo-hexanoate (72) & $\mathrm{C}_{9} \mathrm{H}_{16} \mathrm{O}_{3}$ & 172 & 2.92 & 9.75 \\
2 & 1,2-Dibromo-2-methyl-propane (73) & $\mathrm{C}_{4} \mathrm{H}_{8} \mathrm{Br}_{2}$ & 216 & 18.27 & 0.01 \\
3 & Benzeneacetic acid, methyl ester (74) & $\mathrm{C}_{9} \mathrm{H}_{10} \mathrm{O}_{2}$ & 150 & 21.58 & 0.02 \\
4 & Azulene (75) & $\mathrm{C}_{10} \mathrm{H}_{8}$ & 128 & 21.72 & 0.09 \\
5 & n-Tetradecane (76) & $\mathrm{C}_{14} \mathrm{H}_{30}$ & 198 & 22.17 & 0.02 \\
6 & Pentadecanoic acid, ethyl ester (77) & $\mathrm{C}_{17} \mathrm{H}_{34} \mathrm{O}_{2}$ & 270 & 36.86 & 0.07 \\
7 & Hexadecanoic acid, methyl ester & $\mathrm{C}_{17} \mathrm{H}_{34} \mathrm{O}_{2}$ & 270 & 39.56 & 0.59 \\
8 & Hexadecanoic acid, ethyl ester & $\mathrm{C}_{18} \mathrm{H}_{36} \mathrm{O}_{2}$ & 284 & 40.86 & 3.12 \\
9 & 10,13-Octadecadienoic acid, methyl ester (78) & $\mathrm{C}_{19} \mathrm{H}_{34} \mathrm{O}_{2}$ & 294 & 42.83 & 2.19 \\
10 & Octadecanoic acid, methyl ester (79) & $\mathrm{C}_{19} \mathrm{H}_{38} \mathrm{O}_{2}$ & 298 & 43.34 & 0.05 \\
11 & Heptadecanoic acid, 16-methyl-, methyl ester (80) & $\mathrm{C}_{19} \mathrm{H}_{38} \mathrm{O}_{2}$ & 298 & 43.37 & 0.04 \\
12 & Ethyl linoleate (81) & $\mathrm{C}_{20} \mathrm{H}_{36} \mathrm{O}_{2}$ & 308 & 44.20 & 4.77 \\
13 & 9-Octadecenoic acid (Z)-, ethyl ester (82) & $\mathrm{C}_{20} \mathrm{H}_{38} \mathrm{O}_{2}$ & 310 & 44.27 & 4.76 \\
14 & Bicyclo[4.3.0]nonane, 3-butyl-4-hexyl-(83) & $\mathrm{C}_{19} \mathrm{H}_{36}$ & 264 & 48.20 & 0.09 \\
15 & 6-Nitroundec-5-ene (84) & $\mathrm{C}_{10} \mathrm{H}_{19} \mathrm{NO}_{2}$ & 185 & 48.24 & 0.06 \\
16 & 1,2-Benzenedicarboxylic acid, bis (2-ethylhexyl) & $\mathrm{C}_{24} \mathrm{H}_{30} \mathrm{O}_{4}$ & 390 & 50.33 & 14.26 \\
& ester (85) & & & & 5.25 \\
17 & Di-n-octyl phthalate (86) & $\mathrm{C}_{24} \mathrm{H}_{30} \mathrm{O}_{4}$ & 390 & 50.41 & 2.03 \\
18 & 1,2-Benzenedicarboxylic acid, diisooctyl ester (87) & $\mathrm{C}_{24} \mathrm{H}_{30} \mathrm{O}_{4}$ & 390 & 50.46 & 0.02 \\
19 & 4,9-Decadienoic acid, 2-nitro-, ethyl ester (88) & $\mathrm{C}_{12} \mathrm{H}_{19} \mathrm{NO}_{4}$ & 241 & 52.06 & 0.01 \\
22 & Octadecane, 1-chloro- (89) & $\mathrm{C}_{18} \mathrm{H}_{37} \mathrm{Cl}$ & 288 & 52.45 & 0.34 \\
26 & 1,2-Benzenedicarboxylic acid, diisononyl ester & $\mathrm{C}_{26} \mathrm{H}_{34} \mathrm{O}_{4}$ & 418 & 53.27 & 0.98 \\
\hline
\end{tabular}

Table 3: IC50 of $A$. tamarii MM11 kojic acid

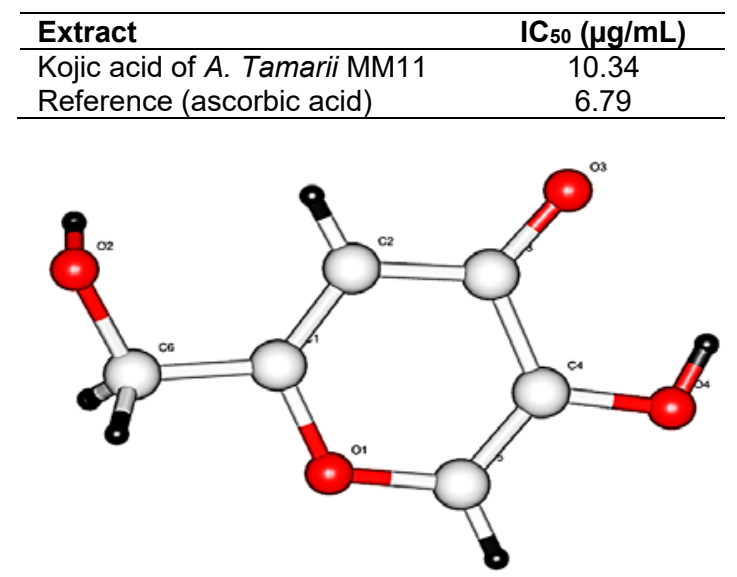

Figure 3: Crystallographic structure of Kojic acid

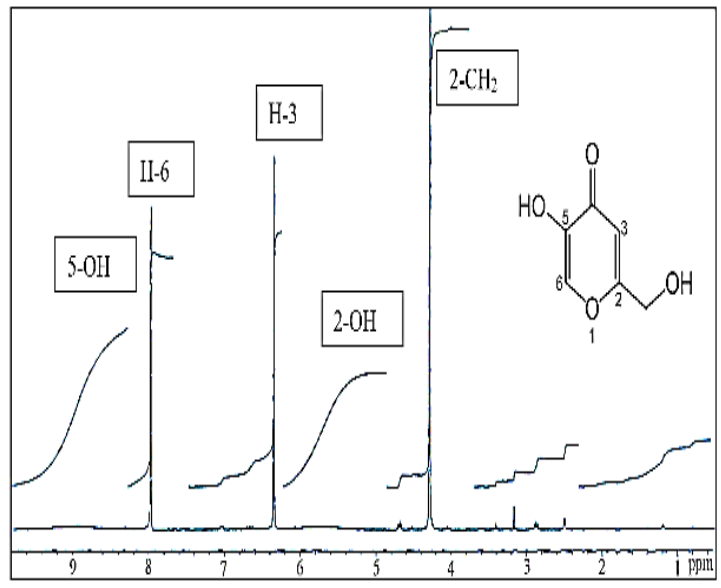

Figure 4: ${ }^{1} \mathrm{H}$ NMR spectrum for kojic acid (1) (DMSOd6, 300MHz). 


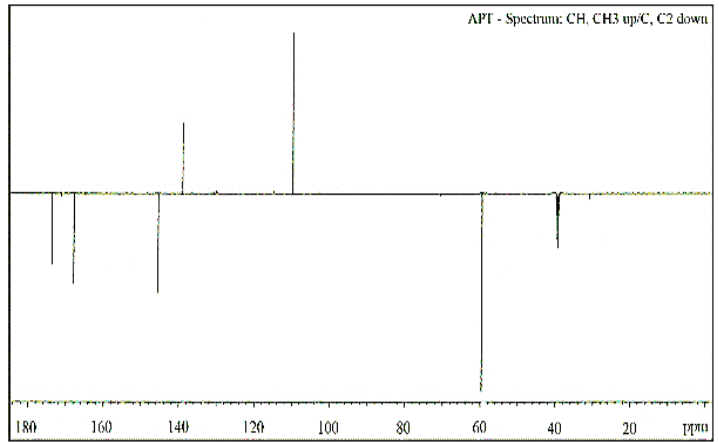

Figure 5: 13 C/APT NMR spectrum for kojic acid (DMSO-d6, 500MHz)

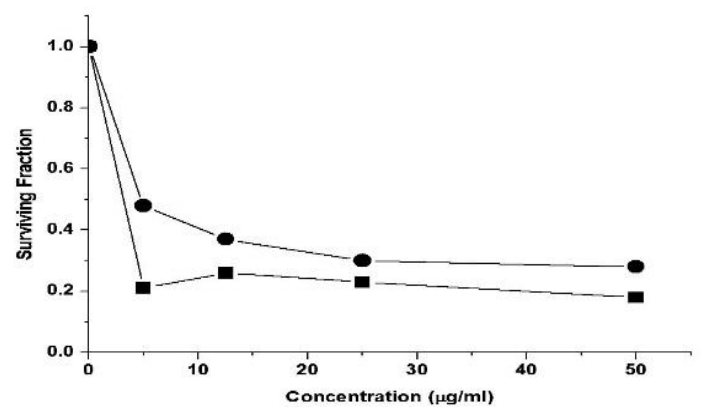

Figure 6: $\mathrm{IC}_{50}$ of Kojic acid from A. tamarii MM11 (•) and DOX ( $\square)$ against HepG-2 cell line.

\section{DISCUSSION}

The great progress in instrumental analysis devices contributed greatly in understand the secondary metabolome map of active fungi. These maps give a platform of secondary metabolites informatics which can be used in many applications. In this article, 100 organic secondary metabolites were identified using GC mass from $A$. tamarii MM11 extract. Generally, $A$. tamarii produces vast types of secondary metabolites including fumigaclavine $A$, aflatoxin, cyclopiazonic acid, speradine $A$ and kojic acid. $A$. tamarii produces a considerable amount of Kojic acid, [13]. Many studies evaluated antimicrobial and antioxidant properties of KOJIC ACID, but only few studies investigated its anticancer activities. In the 1950s, Gerschman et al [14] demonstrated that oxygen-containing free radicals. have hazardous effects on all living cells. Reactive oxygen species (ROS) are endogenous, very active oxygen bearing atoms, which can be divided into enzymatic and nonenzymatic classes [15]. ROS have been believed to be the main cause of various diseases as cancer, sclerosis, Parkinson's, Alzheimer's, immune system ailment, stroke, and others [16]. Antioxidants are the compounds that can neutralize ROS and provide protection against cancer by lowering the peril of tumor development [17].

From the results, purified Kojic acid from $A$. tamarii MM11 gave relatively high DDPH radical scavenging activity. It showed potent antioxidant properties with a very close $\mathrm{IC}_{50}$ to that of the reference ascorbic acid. Kojic acid can be effectively served as nontoxic naturally occurring antioxidant, blocking the action and side effects of many routinely ROS produced during the photodynamic therapy of neoplastic diseases and others such as arteriosclerosis and diabetes [18]. As a promising result, Kojic acid showed highly cytotoxic effects on HepG-2 cells that suggesting strong antitumor effects of Kojic acid against hepatocellular carcinoma. These results were previously observed by another study indicated that Mannich indicated by a study documented that the combination therapy of Mannich base containing ciprofloxacin and Kojic acid structural units showed antitumor activity in HepG-2 [19]. Kojic acid is a potent inhibitor for cellular NF- kappaB activity in different cell types. It is documented that KOJIC ACID has this inhibitory effect in transfectant HaCaT cells, SCC-13 cells and in human keratinocytes. It was found to be more effective than other antioxidants as ascorbic acid and $\mathrm{N}$-acetyl-Lcysteine which suggested that Kojic acid induced anti-melanogenic effect [20]. Previous studies showed that KOJIC ACID also inhibit cell growth of A375 melanoma cells. So, it is used now as a treatment for many types of melanoma [21,22].

There is argument about the effect of Kojic acid administration and DNA mutations. There was a study suggested the ability of Kojic acid to cause mutations in salmonella bacteria [3]. However, other in vivo mammalian studies proved KOJIC ACID as a safe drug at relatively high concentrations that is not significant acute oral toxicant in mice and rats with $\mathrm{LD}_{50}$ value greater than $1 \mathrm{~g} / \mathrm{kg}[6,23]$.

\section{CONCLUSION}

The purified form of Kojic acid isolated from Aspergillus tamarii MM11 shows radical scavenging activity close to that of ascorbic acid. It also exhibits good cytotoxic activity in HepG-2 cell lines. Thus, kojic acid is a potentially safe, natural antioxidant and antitumor agent.

\section{DECLARATIONS}

\section{Acknowledgement}

The authors extend their appreciation to the Deanship of Scientific Research at King Khalid 
University, Abha, KSA for funding this work through Research Groups Program (grant no. R.G.P.1/117/40).

\section{Conflict of interest}

No conflict of interest is associated with this work.

\section{Contribution of authors}

We declare that this work was done by the authors named in this article and all liabilities pertaining to claims relating to the content of this article will be borne by the authors.

\section{Open Access}

This is an Open Access article that uses a funding model which does not charge readers or their institutions for access and distributed under the terms of the Creative Commons Attribution License (http://creativecommons.org/licenses/by/ 4.0) and the Budapest Open Access Initiative (http://www.budapestopenaccessinitiative.org/rea d), which permit unrestricted use, distribution, and reproduction in any medium, provided the original work is properly credited.

\section{REFERENCES}

1. Dewick PM. The acetate pathway: fatty acids and polyketides. Medicinal Natural Products: A Biosynthetic Approach, 3rd Edition; 1997: p. 39-135.

2. Cole RJ, Schweikert MA, Jarvis BB. Handbook of secondary fungal metabolites, 3-volume set; 2003: Academic Press.

3. Carvalho $C$, Santos $R X$, Cardoso $S$, Correia $S$. Doxorubicin: the good, the bad and the ugly effect. Curr Med Chem Des 2009; 16(25): 3267-3285.

4. Yokoo $S$, Masuda $S$, Yonezawa $A$, Terada $T$. Significance of organic cation transporter 3 (SLC22A3) expression for the cytotoxic effect of oxaliplatin in colorectal cancer. Drug Metab Dispos 2008; 36(11): 2299-2306.

5. Bentley R. From miso, sake and shoyu to cosmetics: a century of science for kojic acid. Nat Prod Rep 2006; 23(6): 1046-1062.

6. Burdock GA, Soni MG, Carabin IG. Evaluation of health aspects of kojic acid in food. Regul Toxicol Pharmacol 2001; 33(1): 80-101.

7. Dowd PF. Toxicological and biochemical interactions of the fungal metabolites fusaric acid and kojic acid with xenobiotics in Heliothis zea (F.) and Spodoptera frugiperda (JE Smith). Pestic Biochem Phys 1988; 32(2): 123-134
8. Mohamad R, Mohamed MS, Suhaili N, Salleh MM. Kojic acid: Applications and development of fermentation process for production. Biotechnol Mol Biol Rev 2010; 5(2): 24-37

9. Ardestani A, Yazdanparast R. Antioxidant and free radical scavenging potential of Achillea santolina extracts. Food Chem 2007; 104(1): 21-29.

10. Vichai V, KirtiKojic acidra K. Sulforhodamine $B$ colorimetric assay for cytotoxicity screening. Nat Prot 2006; 1(3): 1112-1116.

11. McKinstry $H$, Eiland $P$, Pepinsky R. The crystal structure of kojic acid. Acta Crystallo 1952; 5(2): 285-286.

12. Fox A. X-Ray Crystallography of Kojic Acid. Nature 1945; 155: 397.

13. Zhang HC, Ma YM, Liu R, Zhou F. Endophytic fungus Aspergillus tamarii from Ficus carica L., a new source of indolyl diketopiperazines. Biochem Syst Ecol 2012; 45 : 31-33.

14. Gerschman R, Gilbert DL, Nye SW, Dwyer P. Oxygen poisoning and $x$-irradiation: a mechanism in common. Science 1954; 119(3097): 623-626.

15. Nathan $C$, Ding $A$. SnapShot: reactive oxygen intermediates (ROI). Cell 2010; 140(6): 951.

16. Valko $M$, Rhodes $C$, Moncol J, Izakovic M. Free radicals, metals and antioxidants in oxidative stress-induced cancer. Chemico-biological interactions 2006; 160(1): 1 40.

17. Chatterjee $M$, Saluja $R$, Kojic acidnneganti $S$, Chinta $S$. Biochemical and molecular evaluation of neutrophil NOS in spontaneously hypertensive rats. Cell Mol Biol (Noisyle-Grand, France) 2007; 53(1): 84-93.

18. Gomes AJ, Lunardi CN, Gonzalez S, Tedesco AC. The antioxidant action of Polypodium leucotomos extract and kojic acid: reactions with reactive oxygen species. Braz J Med Biol Res 2001; 34(11): 1487-1494.

19. Fu Y, Yang Y, Zhou S, Liu Y. Ciprofloxacin containing Mannich base and its copper complex induce antitumor activity via different mechanism of action. Inter $\mathrm{J}$ Oncol 2014; 45(5): 2092-2100.

20. Moon KY, Ahn KS, Lee J, Kim YS. Kojic acid, a potential inhibitor of NF-KB activation in transfectant human HaCaT and SCC-13 cells. Arch Pharm Res 2001; 24(4): 307-311.

21. Cheng SL, Liu RH, Sheu JN, Chen ST. Toxicogenomics of kojic acid on gene expression profiling of $A 375$ human malignant melanoma cells. Biol Pharm Bull 2006; 29(4): 655-669

22. Wang JJ, Wu CC, Lee CL, Hsieh SL. Antimelanogenic, Antioxidant and Antiproliferative Effects of Antrodia camphorata Fruiting Bodies on B16-F0 Melanoma Cells. PLoS ONE 2017; 12(1): e0170924.

23. Abdullah J. Study of using kojic acid as anticancer in different concentration to treatment cancered rats Rattus norvegicus by cyclophosphamide. J Med Res 2017; 1(1): 6-12 